\title{
Supergravity with broken Lorentz invariance
}

\author{
Arthur Marakulin ${ }^{1, \star}$ and Sergey Sibiryakov ${ }^{1,2,3, \star \star}$ \\ ${ }^{1}$ Institute for Nuclear Research of the Russian Academy of Sciences, Moscow, Russia \\ ${ }^{2}$ CERN Theory division, Geneva, Switzerland; \\ ${ }^{3}$ Institut de Physique, EPFL, Lausanne, Switzerland
}

\begin{abstract}
The supersymmetric extension of the Lorentz violating Einstein-aether theory of gravity is considered. The most general Lagrangian of the linearized Einstein-aether supergravity is constructed using the superfield formalism. The constraints imposed by supersymmetry on the parameters of the theory are obtained.
\end{abstract}

\section{Introduction}

Incompatibility of the principles of quantum field theory with general relativity is one of the most important problems in modern theoretical physics. A potential way out of this situation consists in restricting the domain of validity of some basic postulates of general relativity and abandoning them at high energy scales. A promising approach to quantization of gravity based on abandoning the Lorentz invariance has been proposed by Horava [1]. The low-energy limit of the Horava theory, called khrono-metric model, presents a special case of the Einstein-aether gravity [2]. In the latter model violation of the Lorentz invariance is described by the time-like vector field $u^{m}$ with unit norm $\left(u^{m} u_{m}=-1\right)$ called aether that minimally couples to the Einstein-Hilbert action for gravity,

$$
\begin{aligned}
S=S_{G R}-\frac{1}{2} \int d^{4} x \sqrt{-g} & \left(c_{1}\left(\nabla_{n} u_{m}\right)^{2}+c_{2}\left(\nabla_{m} u_{m}\right)^{2}\right. \\
& \left.+c_{3} \nabla_{n} u_{m} \nabla^{m} u^{n}-c_{4} u^{r} u^{s} \nabla_{r} u_{m} \nabla_{s} u^{m}\right) .
\end{aligned}
$$

A phenomenologically acceptable theory with Lorentz violation in the ultraviolet must incorporate a mechanism that ensures emergence of Lorentz invariance at low energies. One of the possible mechanisms is based on non-relativistic supersymmetry [3]. It has been shown [4] that in the supersymmetric extension of the Einstein-aether theory based on chiral vector super-aether field interactions between the aether and the fields of the Standard Model are suppressed.

In this work we consider the coupling of supersymmetric aether to supergravity. This cannot be realized within the minimal $N=1$ supergravity which does not allow to define a chiral aether vector superfield $\left(\bar{\nabla}_{\dot{\alpha}} U^{a}=0\right)$ : anti-commutator of two supercovariant derivatives with spinor indices acting on a vector superfield does not vanish prohibiting to impose the chirality condition. This problem is resolved in the non-minimal formulation of the $N=1$ supergravity with Ziegel parameter $n=-1$ [5].

\footnotetext{
${ }_{\star}^{\star}$ e-mail: marakulin@physics.msu.ru

$\star \star$ e-mail: sergey.sibiryakov@ cern.ch
} 
We use the latter formulation to construct a superfield Lagrangian for the aether field coupled to linearized gravity and prove that it is unique, up to the choice of a single parameter.

\section{Superfield formalism}

Non-minimal linearized $n=-1$ supergravity is formulated [5] in terms of real superfield $V^{a}$ and so-called linear compensator $\Gamma\left(\bar{D}^{2} \Gamma=0\right)$ as a gauge theory with the action

$$
S_{S G}=\int d^{8} z\left(\frac{1}{4}\left(\left(\partial_{k} V_{b}\right)^{2}-\left(\Delta_{k} V_{b}\right)^{2}\right)+\Delta_{k} V_{k}(\Gamma+\bar{\Gamma})+i \partial_{k} V_{k}(\Gamma-\bar{\Gamma})-\Gamma^{2}-\bar{\Gamma}^{2}-\Gamma \bar{\Gamma}\right),
$$

where $\Delta_{k} V_{n}=\frac{1}{4} \bar{\sigma}_{k}^{\dot{\alpha} \alpha}\left[\bar{D}_{\dot{\alpha}}, D_{\alpha}\right] V_{n}$. The action is invariant under super-gauge transformations

$$
\begin{gathered}
\delta V_{\alpha \dot{\alpha}}=\bar{D}_{\dot{\alpha}} L_{\alpha}-D_{\alpha} \bar{L}_{\dot{\alpha}} \\
\delta \bar{\Gamma}=\frac{1}{4} D^{\alpha} \bar{D}^{2} L_{\alpha}
\end{gathered}
$$

We construct linearized Einstein-aether supergravity theory in terms the latter superfields and the chiral vector super-aether field which is considered as consisting of the real vacuum and small perturbation: $U^{a}=W^{a}+\mathcal{U}^{a}$. The super-aether field gauge transformation

$$
\delta_{L} \mathcal{U}_{a}=\frac{1}{4}\left(\sigma_{a b}\right)_{\beta}^{\gamma} D_{\gamma} \bar{D}^{2} L^{\beta} W^{b}+\text { h. c. }
$$

is consistent with the chirality condition. A straightforward but tedious calculation yields the most general Lagrangian invariant under gauge transformations with one free parameter $C$ :

$$
\begin{aligned}
& L=\frac{1}{4}\left(\left(\partial_{k} V_{b}\right)^{2}-\left(\Delta_{k} V_{b}\right)^{2}\right)+\Delta_{k} V_{k}(\Gamma+\bar{\Gamma})+i \partial_{k} V_{k}(\Gamma-\bar{\Gamma})-\Gamma^{2}-\bar{\Gamma}^{2}-\Gamma \bar{\Gamma} \\
&+C\left\{\mathcal{U}_{a} \overline{\mathcal{U}}^{a}-W^{a} W^{c}[\right. \frac{3}{16} \eta_{a c}\left(\left(\Delta_{k} V_{b}\right)^{2}-\left(\partial_{k} V_{b}\right)^{2}\right)-\frac{1}{4} \eta_{a c}\left(\left(\Delta_{b} V_{b}\right)^{2}-\left(\partial_{b} V_{b}\right)^{2}\right) \\
&\left.\left.+\Delta_{a} V_{c}(\Gamma+\bar{\Gamma})+i \partial_{a} V_{c}(\Gamma-\bar{\Gamma})+\frac{1}{8} \eta_{a c}\left(\Gamma^{2}+\bar{\Gamma}^{2}\right)-\frac{1}{4} \eta_{a c} \Gamma \bar{\Gamma}\right]\right\}
\end{aligned}
$$

This parameter is a coupling constant of the super-aether to supergravity.

\section{Component expressions}

In the real world supersymmetry must be broken. As shown in [4], a soft breaking of supersymmetry gives mass to the imaginary component of the super-aether, whereas its real part remains massless. At low energies, the theory is described by the action (1), where the parameters $c_{i}, i=1,2,3,4$, however, are constrained by supersymmetry. To find these constraints, we write the bosonic part of the Lagrangian (6) in component fields. We use the bosonic parts of the gravitational superfield in the Wess-Zumino gauge [5],

$$
\left.V^{m}(x)\right|_{\mathrm{bos}}=\theta \sigma_{a} \bar{\theta} e_{m}^{a}(x)+\theta^{2} \bar{\theta}^{2} A^{m}(x),
$$

where $e_{m n}$ is the symmetrized vielbein; the linear compensator in terms of the "left" coordinates $\left(y^{m}=x^{m}+i \theta \sigma^{m} \bar{\theta}, \theta^{\alpha}\right)$

$$
\left.\Gamma(y)\right|_{\mathrm{bos}}=\theta^{2} B(y)+\theta \sigma^{a} \bar{\theta}\left(v_{a}(y)+i \mu_{a}(y)\right)
$$


and the aether superfield

$$
\left.\mathcal{U}_{b}(y)\right|_{\text {bos }}=v_{b}(y)+\theta^{2} G_{b}(y)+\theta \sigma^{k} \bar{\theta} f_{b k}(y)
$$

where $w_{a}$ is the aether vacuum expectation value, $v_{a}$ is the first order aether perturbation, and

$$
f_{b k}=w_{c} A_{p} \operatorname{tr}\left(\sigma_{b c} \sigma_{p k}\right)+i w_{c} \partial_{m} e_{n p} \operatorname{tr}\left(\sigma_{k} \bar{\sigma}_{p} \sigma_{b c} \sigma_{n m}\right)+w_{c}\left(v_{p}+i \mu_{p}\right) \operatorname{tr}\left(\bar{\sigma}_{b c} \bar{\sigma}_{k p}\right) .
$$

Expanding in the Grassmann coordinates we obtain the bosonic part of the Lagrangian in components. Integrating out the auxiliary fields we reduce to the linearized Lagrangian of the Einstein-aether theory

$$
\begin{aligned}
L_{b o s}=\left(1+\frac{C}{4}\right)\left(\frac{1}{2}\left(\partial_{k} e\right)^{2}-\right. & \left.\frac{1}{2}\left(\partial_{k} e_{m n}\right)^{2}+\partial_{m} e_{m p} \partial_{n} e_{n p}-\partial_{m} e_{m p} \partial_{p} e\right) \\
+C\left(-\left(\partial_{k} v_{b}\right)^{2}+2 w_{a} \partial_{c} v_{b}\left(\partial_{a} e_{b c}-\partial_{b} e_{a c}\right)\right. & \left.-w_{a} w_{c}\left(\partial_{p} e_{a k}-\partial_{a} e_{p k}\right)\left(\partial_{p} e_{c k}-\partial_{c} e_{p k}\right)\right),
\end{aligned}
$$

where $e_{m n}$ is the symmetrized vielbein, $w_{a}$ is the aether vacuum expectation value and $v_{a}$ is the first order aether perturbation. By comparing with the linearized form of the action (1) one finds that supersymmetry fixes all but one coupling constants in this action to zero: $c_{1}=4 C /(4+C), c_{2,3,4}=0$.

\section{Discussion}

In this paper the supersymmetric extension of the Lorentz violating Einstein-aether theory of gravity was considered. The most general Lagrangian of the linearized Einstein-aether supergravity was constructed using the superfield formalism. The constraints imposed by supersymmetry on the theory parameters are obtained.

Modern experimental tests provide tight bounds on the parameters of Lorentz violation. In general, the Einstein-aether model gives rise to non-zero values of the post-Newtonian parameters $\alpha_{1,2}$ that can be expressed in terms of $c_{i}$ [2]. These are constrained from observations in the Solar System: $\left|\alpha_{1}\right| \lesssim 10^{-4},\left|\alpha_{2}\right| \lesssim 4 \times 10^{-7}$ [6]. In the supersymmetric model $c_{1} \neq 0, c_{2,3,4}=0$, so the constraints translate into a bound on $c_{1}:\left|c_{1}\right| \lesssim 10^{-7}$.

The work is supported by the RFBR grant 14-02-31429.

\section{References}

[1] P. Horava, Physical Review D, 79, 8, 084008 (2009).

[2] T. Jacobson, PoS QG-PH, 020 (2007).

[3] S. Groot Nibbelink and M. Pospelov, Phys. Rev. Lett. 94, 081601 (2005).

[4] O. Pujolas and S. Sibiryakov, JHEP, 1201, 062 (2012).

[5] I. L. Buchbinder and S. M. Kuzenko, Ideas and methods of supersymmetry and supergravity, or A walk through superspace. (Institute of Physics Pub., 1998).

[6] B. Z. Foster, and T. Jacobson. Physical Review D, 73, 6, 064015, 1-9 (2006). 